\title{
Multivariate Flood Risk Assessment of the Unplanned Semi- Urban Region by Incorporating Flood Hazard, Vulnerability, and Exposure
}

Sachin Bhere ( $\nabla$ studentsachinbhere@gmail.com )

Indian Institute of Technology Bombay https://orcid.org/0000-0003-0670-4941

M. Janga Reddy

Indian Institute of Technology Bombay

\section{Research Article}

Keywords: Flood risk, Vulnerability, Exposure, Hazard, Kulgoan-Badlapur Municipal Council, Ulhas River, vaccination, vaccines

Posted Date: January 28th, 2022

DOl: https://doi.org/10.21203/rs.3.rs-1286315/v1

License: (c) (i) This work is licensed under a Creative Commons Attribution 4.0 International License.

Read Full License 


\section{Multivariate flood risk assessment of the unplanned semi- urban region by incorporating flood hazard, vulnerability, and exposure Sachin Bhere ${ }^{1^{*}}$ and M. Janga Reddy ${ }^{1,2}$ ${ }^{1}$ Department of Civil Engineering, Indian Institute of Technology Bombay, Mumbai.

Corresponding Author

Sachin Bhere*

Address: Department of Civil Engineering, Indian Institute of Technology Bombay, Powai,

Mumbai, Maharashtra 400076, India.

Mobile No: +919768928318

Email: 204040025@iitb.ac.in 
The mapping of flood risk is important to identify areas at risk and to improve flood disaster management and preparedness. Flood risk is often expressed as the product of the hazard and the probable consequences which is determined in terms of direct damages by assessing flood vulnerability and exposure. This study aims to develop the flood risk assessment (FRA) framework for the dense semi-urban region by incorporating flood hazard, topographic and socio-economic vulnerability, along with exposure, which is calculated by considering housing conditions and classification of the damages by different land use and land cover classes. The FRA at the municipal level is challenging due to the spatial resolution of social, economic, and medical indicators therefore this study attempts to map the flood risk of the semi-urban region where the different zones and housing communities are intertwined due to a lack of town planning. This FRA framework is applied to the KulgoanBadlapur Municipal Council (KBMC) located at the Ulhas Riverbank, a west-flowing river in Maharashtra, India. The city is located at the riverbank, which receives more than $2000 \mathrm{~mm}$ rainfall annually, and as most growing industries and businesses depend on the river itself, the risk associated with flood increases exponentially. The study shows that the spatial distribution of the flood risk is higher in the wards which are densely populated and near the river stream. Despite low population and assets, some neighborhoods are highly susceptible to flood due to their topographical conditions. Over the years, the population has been increasing in the neighborhoods due to the new real estate projects, which will make them more vulnerable to floods, and the overall risk is increasing. Study shows that the $82 \%$ of the area of Valavli and Manjarli ward comes under high flood risk due to high topographic vulnerability and exposure. Similarly the few parts of Industrial zone also comes under the high risk because of its location near river bank. The wards like Kulgaon comes under medium flood risk despite its high socio-economic vulnerability and high exposure which proves that the flood risk is majorly depends on the flood inundation. The exposure-based flood risk assessment will help to frame a more practical and reasonable evaluation of risk for growing urban and industrial zones.

Keywords: Flood risk; Vulnerability; Exposure; Hazard; Kulgoan-Badlapur Municipal Council; Ulhas River.

\section{INTRODUCTION}

A flood is defined as the water overflow from river banks to the adjoining area. The damage caused by floods in terms of loss of life, property, and economic loss is all too well known (Merz et al. 2010; Nofal and van de Lindt 2020; Sen, Dutta, and Laskar 2021; Svetlana, Radovan, and Ján 2015). Floods have a huge impact than any other disaster event and have a high destructive potential to alter the ecology, and it can cause social and economic damages. Floods are one of the disastrous extreme hydrological events responsible for the thousands of casualties worldwide and billion dollars of economic damage (Komolafe, Herath, and Avtar 2018; Mokhtari, Soltani, and Mousavi 2017)With an increase in population every year, more and more flood-prone areas are occupied for residential, agricultural, and industrial activities. Under normal conditions, the area was used for floodwater storage now has become build-up areas, leading to frequent flooding of these areas and the possibility of large-scale economic damages (Grigg 2020; Koks et al. 2015). In the past, different studies expressed the flood risk as a function of flood hazard and vulnerability which analyzes the intensity of the flood and the damage caused to the assets (Ali, Bajracharya, and Koirala 2016; assessment to a multi-dimensional risk assessment considering the factors such as social and economic 
vulnerability(Bengal, Chakraborty, and Mukhopadhyay 2019; Ghosh and Kar 2018; Nguyen et al. 2021; Sahoo and Sreeja 2017; Tripathy et al. 2020a). The advances in the capacity of computational resources help to incorporate multiple parameters responsible for the flood risk (Doorga et al. 2022; Monteil et al. 2022). In single-dimensional FRA, the flood hazard is considered as one of the following factors like rainfall intensity, flood inundation, flood depth, and rising sea level in coastal flooding. The multivariable FRA depends on the flood hazard, vulnerability, and exposure, structural and non-structural measures as flood preparedness. Along with the hazard, flood vulnerability is essential in assessing the possible damage due to flood events (Bengal, Chakraborty, and Mukhopadhyay 2019; Franci et al. 2016; Kabenge et al. 2017). The socio-economic conditions can be considered to evaluate the vulnerability and compute the direct and indirect damages. The flood exposure still hasn't been incorporated well in the flood risk framework, maybe because it requires combining the quantitative and qualitative data(Imran et al. 2019; Kittipongvises et al. 2020; Mohanty, Mudgil, and Karmakar 2020).

The FRA for the unplanned semi-urban region is challenging due to homogenous socio-economic conditions, which makes it difficult to divide the municipal council into different zones. The study attempts to classify the wards of the municipal council based on various indicators which are responsible for flood vulnerability and exposure. In this study, the flood risk of the Kulgoan Badlapur municipal council (KBMC), Maharashtra, India is assessed by incorporating flood exposure with socio-economic and topographic vulnerability and flood hazard. The KBMC is located near Western ghat, India, and The Ulhas River flows through the KBMC, which is the home of around 18.4 million people, according to the 2011 census. Due to its location in the Mumbai metropolitan region (MMR) and the industrial zone, the population is increasing. As the city is located at the river bank and most growing industries and businesses depend on the river itself, the flood vulnerability increases exponentially. For FRA, several studies majorly focused on the flood hazard and vulnerability, which fails to assess the possible direct and indirect damages to the infrastructure and industrial zone. Therefore, the main objective of this study is to incorporate the exposure associated with the industrial zone and infrastructure into the risk assessment framework along with socio-economic vulnerability. Along with the main objective following are the some objectives of the study:

- This study aims to develop the FRA framework for the unplanned semi-urban region by incorporating flood hazard, socio-economic, topographic, and medical vulnerability along with infrastructural exposure.

- To develop the flood risk map at ward level for municipal councils to allocate the resources for the flood mitigation.

- To assess the risk involved with the different resources in terms of direct probable damages by including the flood exposure based on the different land use and zones in municipal council.

The flood hazard is considered as the flood inundation simulated using the HEC-RAS model. The vulnerability is calculated by considering different topographic and socio-economic parameters using Analytical Hierarchy Process (AHP), and the exposure is calculated by considering plinth height, road network density, and type of buildings. The following sections discuss the proposed framework for flood risk assessment, and the subsequent section presents the application of the proposed FRA framework for the KBMC region, and the corresponding results are discussed focusing on evaluating different flood risk parameters for each ward of the KBMC area. 


\section{METHODOLOGY}

The risk is expressed as the expected loss of life, property damage, disruption of economic activity, environmental damage, etc (Barrett, Steinbach, and Addison 2021). This provides the base for the multidimensional flood risk assessment in which the consequences in terms of expected losses are essential to calculate and interpret flood risk effectively along with the flood hazard. This study incorporates topographic, socio-economic vulnerability, and exposure for semi-urban unplanned municipal council and Figure 1 shows the flow chart of the proposed framework.

The flood hazard is calculated by classifying the risk level of flood inundation, and the assessment of consequences are divided into vulnerability and exposure. The vulnerability is determined based on the combined index of topographic factors, socio-economic factors, and medical indicators similarly the exposure is calculated using two indicators, viz.,indicator based on road networks across cities and housing types, and the other indicator based on the degree of importance of the land use and land cover classes. Finally the FRA can be interpreted mathematically as the product of flood hazard, vulnerability, and exposure.

The flood hazard is calculated considering the flood inundation extent corresponding to different return periods (50-year, 100-year, and 200-year). The flood magnitude of the various return period is calculated using Intensity-Duration-Frequency (IDF) curve. The flood hazard assessment in various studies involved the determination of the flood inundation, estimating the peak discharge and associated water levels, along with the probability of the extreme rainfall or discharge (Cançado et al. 2008; Díez-Herrero and Garrote 2020; Ganguli and Reddy 2013; Koks et al. 2014; Kron 2002; Molinari et al. 2019; Tripathy et al. 2020b; Wang et al. 2019). To estimate the flood hazard, few studies proposed the multi-criteria methods, which calculate the hazard based on the different factors associated with it. This type of calculation is more suitable for the spatial analysis of the hazard, where the observed discharge and associated water levels data are not available. Figure 2 shows the flow chart of methodology for mapping of the flood hazard.

For calculating the flood extent, steady flow hydraulic modeling is performed using the HEC-RAS model. The HEC-RAS model uses energy, continuity, and momentum equations with an iterative procedure using the standard step method (USACE 2016). The HEC-RAS model computes the flood water elevation by taking inputs of the geometrical data and discharge values for different rainfall intensities, and the discharge values at all reaches, junctions, and watersheds are computed using the hydrological model, HEC-HMS (Feldman 2000). The HEC-HMS uses the SCS-CN method for calculating the runoff volume in which the rainfall data and the CN grid are required as the model's input. The 50 years, 100 years, and 200 years rainfall intensities are calculated using Intensity-Duration-Frequency (IDF) curves (Kothyari and Garde 1992) in which the hourly rainfall data of Indian Meteorological Department's (IMD) is used. After calculating the rainfall intensities, the values are used as an input in the calibrated HEC-HMS model. The discharge values are incorporated into the HEC-RAS model to estimate the flood inundation extent for 50 years, 100 years, and 200 years which then divided into different hazard zones. After calculating the flood hazard the flood vulnerability is calculated based on two aspects: The city's physical and topographical conditions and the socio-economic condition to bear the shocks after flood events or to understand the resilience towards floods. Figure 3 shows the flow chart for determining the high and low vulnerable zones in the municipal council. 
To calculate the topographic vulnerability, factors like elevation, slope, distance from the river, drainage density, flow accumulation, topographic wetness index (TWI), Stream power index (SPI), and curvature are considered. The elevation is the factor that influences highly as compared to the other parameters. The low elevation area has a high probability of getting flooded as compared to the high elevation. The distance from river and drainage density are key parameters to understand the extent and time for the flood water to reach into the city. The topographic wetness index (TWI) is calculated to understand the probable flood inundation regions in the basin which is calculated using Moore's equation (Moore, Grayson, and Ladson 1991). Other factors like flow accumulation, curvature, and stream power index (SPI) also help to determine the critical regions vulnerable to floods. To calculate the combined topographic vulnerability index, the Analytical Hierarchy Process (AHP) is used.

The AHP method introduced by (Saaty 1987) which uses the pairwise comparison matrix to calculate the weightage of each parameter to establish the vulnerability map. The comparison between different parameters is made by assigning the numerical values to each component based on the relevance and significance (Bengal, Chakraborty, and Mukhopadhyay 2019; Chen, Yeh, and Yu 2011; Danumah et al. 2016; Ghosh and Kar 2018; Hu et al. 2017; Lin, Wu, and Liang 2019; Ramkar and Yadav 2021). Table 1 shows the pairwise comparison of different topographic parameters. For example, ' 1 ' is for the equally significant, 3 for moderately more significant, 5 for strongly more significant, and 7 for very strongly more significant. And 2, 4, and 6 are the intermediate scores. Table 2 shows the normalized weightage of each topographic parameter.

The steps involved in the AHP procedure for calculating the weightages is given below:

1. Identify the important factors and/or parameters in the flooding problem.

2. Arranging the parameters into hierarchical order based on each parameter's influence on the flood vulnerability.

3. According to the relevance of the factors, the numerical value is assigned from 1 to 7 .

4. A comparison matrix is built, and the eigenvector computes the normalized weights.

The comparison matrix is built with the diagonal elements being equal to 1 , using the following expression:

$A=\left(a_{i j}\right)_{m * n}=\left[\begin{array}{cccc}a_{11} & a_{12} & \ldots & a_{1 n} \\ a_{21} & a_{22} & \ldots & a_{2 n} \\ \vdots & \vdots & & \vdots \\ a_{n 1} & a_{n 2} & \ldots & a_{n n}\end{array}\right]$,

$a_{i i}=1, a_{i j}=\frac{1}{a_{j i}}, a_{i j} \neq 0$

The consistency of the AHP method is assessed using consistency ratio (CR). Following is the expression for CR (Saaty 1987):

$C R=\frac{\text { Consistency Index }(C I)}{\text { Random Index }(R I)}, \quad \mathrm{CR}<0.10$ is acceptable

Where, $C I=\frac{\lambda \max -n}{n-1}, \lambda \max =$ Principal eigenvalue of the matrix

The Random Index (RI) is determined by the number of parameters in the comparison matrix. The value of RI is referred from the table given in Saaty's 1980 work on the AHP. Each topographic factor is divided into five categories, and the sub-criteria AHP analysis is done for every element to neglect the effect of the different units. Table 3 presents the sub-criteria pairwise comparison matrix of each parameter.

Along with the topographic vulnerability, the socio-economical plays an essential role in understanding indirect flood damages. The factors such as total population, population density, household income, and medical 
indicator which is based on the percentage of the people vaccinated against the flu, etc., are considered as key factors to understand the spatial distribution of the vulnerability. The choice of indicator for the vulnerability depends on the demographic data availability. Some indicators are positive in terms of flood vulnerability, which increases the vulnerability with an increase in the values, for example, the total population across different wards, population density, and the number of houses. Some indicators help to reduce the flood vulnerability as the percentage of vaccination of the dependant population, interprets the region's health condition. High vaccination percentage indicates the low vulnerability of the people to water-borne diseases due to floods. The combined vulnerability index is then calculated by assigning a weight to each factors based on how they influence the flood vulnerability, more or less. Using the standardization approach, the parameters are converted into a scale from maximum to minimum by assigning 1 to 0 . Considering $\mathrm{V}_{\mathrm{i}}$ as each indicator's pixel value, $\mathrm{V}_{\max }$, and $\mathrm{V}_{\min }$ are the maximum and minimum values of the parameter, the standardized parameters $\left(\mathrm{V}_{\text {std }}\right)$ for negative and positive indicators are estimated by following formulas (Tripathy et al. 2020b).

$$
\begin{aligned}
V_{s t d} & =\frac{V_{i}-V_{\min }}{V_{\max }-V_{\min }}(\text { Negative Indicator }) \\
V_{\text {std }} & =\frac{V_{\max }-V_{i}}{V_{\max }-V_{\min }}(\text { Positive Indicator })
\end{aligned}
$$

The combined vulnerability index ( $\mathrm{Vul}$ ) is calculated by assigning the weightages $w_{l}$ and $w_{2}$ to the topographic vulnerability index $\left(\mathrm{Vul}_{\text {topo }}\right)$ and socio-economical vulnerability index $\left(\mathrm{Vul}_{\mathrm{SE}}\right)$, respectively.

$$
V u l=W_{1} * V u l_{\text {topo }}+W_{2} * V u l_{S E}
$$

Along with the vulnerability, the exposure is also incorporated for calculating the flood risk. The exposure helps to narrow down the vulnerability that can predict the state of direct damages due to floods.

Exposure is as significant as calculating flood hazard and vulnerability for analysing the flood risk. The exposure is calculated by considering two major factors. The first factor deals with the type of land use that comes under the flood extent based on the degree of importance, and the second is based on the building and infrastructure condition. Figure 4 shows the flowchart for calculating the exposure.

Many unplanned municipal corporations in India have different zones which are intertwined like industrial zones, built-up, plantation, forest, and at some places, agricultural land too. Each land uses have a different level of importance based on its operation, economic output, and the number of people associated with it. Considering all factors, the degree of importance is assigned to land use and land cover classes ranging from 0 to 1 . For example, the Industrial zone is considered high exposure to flooding due to possible severe direct damages, and therefore the high degree of importance is assigned to it. Table $\mathbf{4}$ shows the degree of importance $\left(D_{i}\right)$ to each land use and zones.

The second component of the exposure is the exposure of buildings and infrastructure $\left(\mathrm{E}_{\mathrm{BI}}\right)$. It is calculated by considering the building's usable plinth height $\left(\mathrm{H}_{\mathrm{p}}\right)$, type of building (Residential Complex, Single residential building, industrial building units, small houses, other institutional buildings), and road density $\left(\mathrm{R}_{\mathrm{d}}\right)$. The attribute table is created for each building and infrastructure, and based on the above factors, the combined exposure value is calculated. For plinth height $\left(\mathrm{H}_{\mathrm{p}}\right)$, the buildings are identified with ground-floor apartments and ground-floor stilt parking. The buildings with ground-floor apartments are considered to have high exposure to the flood as compared to the stilt parking facilities. Most of the residential complexes have a usable floor above 5 meters from ground level, which is less exposed to the flood. The single residential buildings with ground-floor apartments have high exposure value. Small individual houses are also considered under high flood 
exposure. Compared to each pixel's 100-year flood depth $\left(\mathrm{D}_{100}\right)$, the apartment's height or usable floor height is calculated as corrected plinth height $\left(\mathrm{H}_{\mathrm{PC}}\right)$. The standardization is done, considered as 'Exposure index for plinth' $\left(\mathrm{EI}_{\mathrm{P}}\right)$, which is assigned to each building ( 0 to 1 , for low exposure to high exposure). The formulas are given below:

$$
\begin{aligned}
H_{P C} & =\frac{\text { Plinth height }\left(H_{p}\right)-D_{100}}{D_{100}} \\
E I_{P} & =\frac{\operatorname{Max}\left[H_{P C}\right]-H_{P C}}{\operatorname{Max}\left[H_{P C}\right]-\operatorname{Min}\left[H_{P C}\right]}
\end{aligned}
$$

After calculating the exposure associated with the plinth height, the exposure index for building $\left(\mathrm{EI}_{\mathrm{B}}\right)$ based on the type of building and amenities are determined. The numerical value of 0.25 is assigned to the residential complex, 0.5 is assigned to single residential buildings and industrial buildings, and 0.75 is for single small houses based on the level of exposure. Similarly, the exposure index for the road $\left(\mathrm{EI}_{\mathrm{R}}\right)$ is calculated based on the road network and density. The standardization is done for each pixel based on the road density's maximum and minimum value $\left(\mathrm{R}_{\mathrm{d}}\right)$. The higher road density leads to high flood exposure and vice versa. The following formulas give the exposure index for the road $\left(\mathrm{EI}_{\mathrm{R}}\right)$ and total exposure value for building and infrastructure:

$$
\begin{aligned}
E I_{R} & =\frac{\operatorname{Max}\left[\mathrm{R}_{d}\right]-\mathrm{R}_{d}}{\operatorname{Max}\left[\mathrm{R}_{d}\right]-\operatorname{Min}\left[\mathrm{R}_{d}\right]} \\
E_{B I} & =\frac{1}{3}\left(E I_{P}+E I_{B}+E I_{R}\right)
\end{aligned}
$$

Exposure is the mean of values of the degree of importance for land use, and exposure associated with building and infrastructure $\left(\mathrm{E}_{\mathrm{BI}}\right)$.

$$
\text { Exposure }=\frac{\mathrm{D}_{i}+E_{B I}}{2}
$$

Using the above equation, the spatial distribution of the exposure is calculated across the municipal council. After calculating the flood exposure, the flood risk is calculated using the following formula;

$$
\text { Flood risk }=\text { flood hzard } X \text { flood vulnerability } X \text { flood exposure }
$$

The calculated flood risk represents the flood inundation and the direct damages caused by the inundation for different return periods, and the socio-economic ability of people and communities to mitigate the flood damages. The analysis also includes the infrastructural exposure, including the detailed analysis of plinth height, type of building, and the road network. The flood risk also consists of the damages to the industrial zone by giving a higher degree of importance. The proposed framework for flood risk assessment is applied to a case study of KBMC and a discussion of the results along with data collected and sources are presented in the following sections.

\section{CASE STUDY AND DATA COLLECTION}

The proposed framework is applied to the Kulgoan-Badlapur Municipal Council (KBMC) located at the bank of the Ulhas River, which is a west-flowing river in India. This section discusses the study area and data collected to determine the flood risk across the municipal council. The Ulhas basin is home to millions people in 6 major municipal corporations like Thane, Kalyan-Dombivili, Ulhasnagar, Bhiwandi-Nijampur, Badlapur, and Ambernath, which makes it the most populated district in the country, and any small natural calamity can lead to massive social and economic disaster (Das 2019a; Das and Pardeshi 2018; Kim et al. 2019; Rangari et al. 2019)Flooding is very frequent in the basin due to its location in the high monsoon region. Due to unplanned urbanization and the climate change the 25-year floods are coming at 10-year frequencies, and the Urban 

the flood line of 17.5 Meters is crossed thirteen times from the year 1991 at the KBMC observation point.

\subsection{DATA COLLECTION}

The flood risk is calculated by determining the flood hazard, its associated topographic and socio-economic vulnerability, and flood exposure. The flood hazard is estimated based on the inundation corresponding to different return periods using hydrological and hydraulic modeling. To calculate the rainfall intensity for different return periods, the Indian Meteorological Department's (IMD) rainfall data over the Ulhas river Basin is used. The watershed is delineated using the ArcHydro tool for hydrological modeling by inputting 30-meter resolution SRTM's digital elevation model (DEM) data, the land use, and soil map are required for generating a $\mathrm{CN}$ grid to calculate the losses. The land use and land cover maps are generated using the Landsat data of $30 \mathrm{~m}$ and $100 \mathrm{~m}$ resolution and the soil map is extracted from the FAO's Global soil map. The model is then calibrated and validated using the observed discharge data of the Badlapur hydro-metro station. After calculating the corresponding discharge for various rainfall intensities and return periods, the values are incorporated into the HEC-RAS model. The geographical data is extracted and mapped in the HEC-GeoRAS tool of ArcGIS, including rivers, channels, and cross-sections that form key input data sets to the HEC-RAS model to run the steady flow analysis to determine the flood inundation (flood hazard). For calculating the extent of flood inundation, the Digital Surface Model (DSM) of the Advanced Land Observing Satellite (ALOS) DSM product of 30 meters (AW3D30) is used, which is available on the JAXA web portal as a base map. The vulnerability is calculated considering topographic and socio-economic factors. Topographic vulnerability factors like elevation, slope, topographic wetness index, stream power index, flow accumulation, and curvature are derived from the SRTM's DEM of 30-meter resolution. The other factors, including distance from the river and drainage density, are calculated from the river shapefiles. For socio-economic vulnerability, population density, household income, and medical indicators based on the vaccination data for flu are considered. The population data is downloaded from the World-pop data portal of 100-meter spatial resolution. The population data is available as the people per pixel, which is calculated based on the random forest approach with Remotely-Sensed and Ancillary Data (Stevens et al. 2015). For medical indicators, the vaccination data is used. The data shows the percentage of the children taking vaccines for flu, based on the study conducted on childhood vaccination performed in middle and low-income countries (Utazi et al. 2018). The household income data is collected by the survey of municipal corporations, based on local taxes. For exposure, urban divisions based on the land use data, road network, and condition of the buildings focusing on the plinth height and construction type are considered. The road network and building's shapefile are downloaded from the OpenStreetMap portal developed by the OpenStreetMap foundation and local survey conducted by the KBMC. The application and the results are discussed in the following section for FRA of KBMC.

\section{APPLICATION AND RESULTS}

The proposed methodology is applied to the Kulgaon Badlapur Municipal council (KBMC), considering hazards as flood inundation, socio-economic and topographic vulnerability, and exposure by considering building plinth 
height and different land use and land cover classes based on the degree of importance. At a resolution of 30 meters, the risk index is calculated for each pixel. The KBMC includes Urban, Semi-urban, Rural settlements and different land use classes, like industrial zones, agricultural land, and Built-up land and each of the categories has a different capacity to withstand the damages caused due to floods. This section discusses the findings of the different flood risk factors, viz., flood hazard, vulnerability, and exposure of each ward which helps to understand the spatial distribution of the flood risk in the KBMC.

\subsection{FLOOD HAZARD ESTIMATION}

The flood hazard is calculated by determining the flood inundation for the different return periods of rainfall. The IDF curve is plotted using Gumbel's equation with the help of hourly rainfall data collected from the Indian Meteorological Department (IMD). The KBMC considers the extreme rainfall events of 100-year frequency for identifying flood lines, and due to climate change and land use pattern change, the intensities are changing for different return periods; therefore, the 200 -year rainfall is also considered to determine the flood inundation. Figure 6 shows the intensity and duration of rainfall for different return periods (frequencies). The graph shows the rainfall intensity as $172.04 \mathrm{~mm}, 145.42 \mathrm{~mm}$, and $122.5 \mathrm{~mm}$ for return periods of 200-year, 100-year, and 50-year, respectively. With the IDF curve, extreme rainfall is calculated and used to determine the flow in the channels and inundation area.

After calculating the different intensities of the rainfall, the values are considered as input into the HEC-HMS model to determine the discharge. The HEC-HMS model is calibrated with 30 years of the observed discharge data from Badlapur hydro-meteorological station. The first ten years of observed discharge data are used for calibration of the model, and the performance is evaluated by calculating the coefficient of determination ( $\mathrm{R}$ squared) value. The model shows a good correlation after calibration with the R-square value of 0.734 , which shows a good correlation between the simulated and observed flow. After calibration of the model, the discharge is simulated at different reaches and junctions which is then incorporated into the HEC-RAS model for determining the flood inundation for different return periods.

The flood hazard is the area inundated due to floodwater from the Ulhas River. The hazard is divided into three classes. Figure 7 shows the spatial extent of the flood inundation associated with different return periods. The spatial extent of the 200-year flood is higher than a 50-year flood, but the area that comes under the extent of the 50-year flood boundary will be affected more frequently. Therefore the risk associated with a flood inundated area of 50-years is higher than the 200-years. The map shows the majority of the flood inundation is happening in the Badlapur, Valhivali, and Manjarli ward (central and south-east ward) of the municipal council. The least affected wards are Kulgaon and Belavali (north-west ward), which have a maximum settlement. Due to the increasing demand for residential apartments, the developers are constructing residential complexes in the high flood hazard wards like Badlapur and Manjarli which will increase the overall flood risk. The flood hazard map also shows that some part of the Maharashtra Industrial Development Council (MIDC) area, an industrial zone of the KBMC, is flooded for 100-year flood events. The vulnerability is high in the MIDC area as most industries have manufacturing units and the efficiency of those industries depends on factors like water supply, electricity, transportation, and many others, which can be affected due to the flooding.

\subsection{FLOOD VULNERABILITY ASSESSMENT}

The flood vulnerability is calculated by considering topographic factors and socio-economic factors. The topographic vulnerability is calculated to determine the flood susceptibility. The primary data used for 
calculating the topographic vulnerability is the DEM data. Multiple factors are considered for calculating the topographic vulnerability and the weightage is assigned using the AHP based multi-criteria decision analysis method. Factors including elevation, slope, distance from the river, drainage density, Stream power index, topographic wetness index, and curvature are used to calculate the combined topographic vulnerability. The single index is formed by assigning the hierarchical priority and weightage. Figure 8(A) shows the spatial distribution of topographic vulnerability for the KBMC area. It also shows the topographic vulnerability is highly correlated to the elevation and distance from the river stream as it has higher weightage in the AHP framework. The Central ward, which is parallel to the stream, is more vulnerable than any wards and the Badlapur ward also shows high topographic vulnerability compared to the old settlement, which is planned and developed considering the flood risk. Some region of MIDC also come under the topographically vulnerable region. As the scope of future settlement in the wards like Badlapur and MIDC with high topographic vulnerability, the overall risk of these wards will be high as compared to the other parts of the KBMC.

The total vulnerability is calculated by combining the topographic and socio-economic vulnerability. For socioeconomic vulnerability, the total population, population density, household income, and medical indicators as percentage of vaccination against flu are considered. All the indices are standardized to a scale of 1 to 0 (high vulnerable to low vulnerable) to form the final socio-economic index. Figure 8(B) shows the spatial distribution of the socio-economic index of the KBMC. The wards with a high urban population and built-up land have high vulnerability despite having good economic conditions compared to the other wards of the municipal council. Here the average household income per pixel is considered, and therefore it can be seen that despite high cumulative wealth in semi-urban regions, the average per capita income is less. The socio-economic vulnerability also depends on the population density, which is higher in the Kulgaon, Shirgaon, and Badlapur wards. The population of the industrial zone is underestimated because the census data included only the living or residential population. Therefore, the vulnerability can be seen as lower than the other wards, which is not the actual picture as a lot of people work in three 8-hour shifts, which makes it more vulnerable. The socioeconomic vulnerability can be reduced by adopting effective town planning, and the population across the city can be managed by constructing and promoting effective residential projects across the different wards of the municipal council. The vulnerability can also be reduced by improving medical facilities across the city.

Figure 8(C) shows the combined ward level vulnerability index standardized to the scale at 0 to 1 by combining the topographic and socio-economic vulnerability. The vulnerability represents the topographic and socioeconomic ability to cope with the flood and to develop resilience. The wards with high population density near the stream are under high flood vulnerability, ultimately under high flood risk. The map shows the settlements in Kulgaon and Valivali have high flood vulnerability, and the Badlapur and Shirgoan have medium to high flood vulnerability.

\subsection{FLOOD EXPOSURE ASSESSMENT}

The flood exposure is calculated using two indicators, first the land use, and land cover classes, by assigning the degree of importance and second the exposure associated with the building and infrastructure. The exposure further disintegrates the vulnerability by including certain factors for example the population density or total population in each ward represents the residential population only and therefore, the population in the industrial zone is low in records compared to other wards, which underestimates the vulnerability. Consequently, the exposure is incorporated in the risk framework by classifying further into different land use and land cover 

land cover classes along with their degree of importance. The degree of importance is assigned based on the probable damage caused by the flood to different land use and land cover classes.

395 Figure 9(A) shows that a higher value is assigned to the MIDC ward, an industrial zone along with Kulgaon and Shirgaon ward, which consists of built-up land, and agricultural zones. Another parameter for calculating the exposure is the exposure associated with the building and infrastructure $\left(\mathrm{E}_{\mathrm{BI}}\right)$; the detailed explanation is given in the methodology section. For plinth height $\left(\mathrm{H}_{\mathrm{p}}\right)$, the buildings are identified with ground-floor apartments and ground-floor stilt parking. The buildings with ground-floor apartments are considered to have high exposure to the flood as compared to the stilt parking facilities. Most of the residential complexes have a usable floor above 5 meters from ground level, less exposed to the flood. The single residential buildings with ground-floor apartments have high exposure value. Small individual houses are also considered under high flood exposure. Along with the plinth, the type of building is also considered for calculating the possible damage in terms of exposure. The numerical value of 0.25 is assigned to the residential complex, 0.5 is assigned to single residential buildings and industrial buildings, and 0.75 is assigned for single small houses. Another factor is by considering the road density which is calculated to understand the location of buildings, institutes, and other infrastructures. The high road density interprets the high flood exposure. The road density is calculated using the line density function and then standardized to the scale of 0 to 1 (low exposure to high exposure). Figure 9(B) shows the exposure map associated with the road density. The infrastructural exposure is calculated by taking an average of three infrastructural exposure indicators, which are road density, plinth level, and type of building. The infrastructural exposure indicates the possible direct damages to the buildings, roads, and industrial zones. The infrastructural exposure is then combined with exposure calculated for land use and land cover based on degree of importance. Figure 9(C) shows the spatial distribution of the flood exposure of the KBMC.

From Figure 9(C), it can also be noted that the exposure of the MIDC ward, an industrial zone, is higher than other wards of the municipal council. The exposure in the map indicates the possible direct damage to the infrastructure, buildings, and resources. It shows the high possibilities of damages in the urban wards of the municipal council because of the low plinth height of the buildings, and single ground floor apartments.

\subsection{FLOOD RISK ASSESSMENT}

The flood risk is assessed as the multiplication of flood hazard, vulnerability, and exposure. All the parameters are converted into a scale of 0 to 1 , low risk to high risk. Figure $\mathbf{1 0}$ shows the spatial distribution of the flood risk. It shows that highly populated wards and near the stream are at higher risk. The map shows the generalized relative risk of the KBMC. The high flood risk can be seen in the Valavli and Manjarli ward. About $82 \%$ of the area of these wards comes under very high to high flood risk zone, which is also validated with the information and aid provided by the municipal council to these wards. The Badlpaur, Yeranjad, and Kulgaon also come under high to medium flood risk zone as $38 \%$ of the area of these wards come under high to medium flood risk. The industrial zone MIDC ward also comes under the high to the medium flood risk zone. The direct damage in the industrial zone is higher but still, due to other factors, the overall risk is reduced as the ward have low population density and high plinth of building due to industrial building construction bylaws. The flood risk of the industrial zone is underestimated as the data regarding the actual damage is not available. Due to changes in climate and land-use patterns, the risk is going to increase as the intensity of flood hazards is going to increase. 
The wards like Badlapur and Shirgaon have more barren land, which has the potential for residential and industrial constructions in the future, which will lead to increase in flood risk despite incorporating all the factors which indicate the direct infrastructural damage and socio-economic capability to mitigate the flood, the risk is highly dependent on the flood hazard.

\section{DISCUSSION}

The study presented an effective framework for flood risk assessment of unplanned semi-urban settlements by incorporating infrastructural and industrial damages into the conventional flood risk framework. The flood risk is expressed as the function of flood hazard, topographic vulnerability, socio-economic vulnerability, and exposure. The flood inundation for different return periods is considered as flood hazard. The rainfall intensityduration-frequency (IDF) curves are developed using hourly rainfall data, and the discharge is simulated for the extreme rainfall using the HEC-HMS model. The discharge values are then used to determine the corresponding inundation by HEC-RAS hydraulic modeling. As the discharge data is not available for the reach passing through the municipal council, and the simulated discharge is used to determine the inundation of the flood. In this study using 1D hydraulic modeling, only water spread area is calculated, but the assessment can be improved by considering multivariable flood hazards. The flood depth at various locations and the flow velocity can be used to enhance overall flood hazard mapping, and the damages can be assessed accordingly.

In this study the vulnerability is calculated by considering two parameters, topographic vulnerability and socioeconomic vulnerability. The topographic vulnerability represents the flood susceptibility which is calculated using the Analytical hierarchy process (AHP). The topographic vulnerability is calculated by considering elevation, slope, drainage density, and distance from the river, curvature, stream power index, and topographic wetness index. The weightage is calculated by making a pairwise comparison of each factor and the priority is assigned based on previous research conducted for flood susceptibility (Das 2019b). The topographic vulnerability assessment can be improved by using a fine resolution elevation grid or more parameters responsible for high flow. The socio-economic vulnerability is calculated along with the topographic vulnerability, considering factors like population density, household income, and medical indicators. The accuracy of socio-economic vulnerability indicates the ability of individuals or communities to mitigate the flood. The socio-economic vulnerability depends on the ground data for the population, demographics, and economic condition. The indicator can be improved by using more fine resolution data with different social indicators. The spatial distribution of socio-economic conditions in urban regions is difficult to interpret as the population is very diverse, and the integration of social and economic conditions makes it more complicated to assess the vulnerability of individual households. For the medical indicator the percentage of vaccination against water-borne diseases are considered but more ground surveys and data will help to improve the assessment of socio-economic vulnerability.

The primary economical source of KBMC is the industries located in the MIDC area. The economic vulnerability of the region depends on the exposure of industries to flood, which is more and more inclusive of electronics. The exposure is calculated by considering the industrial zone and the infrastructural condition of the municipal council, along with the infrastructural exposure which is calculated by considering the plinth height 
of buildings and its association with depth of 100-year flood, the road density, and type of buildings. The buildings are divided into high elevated residential buildings, small houses, and industrial buildings. The risk is more to the small houses and the buildings with low plinth height. Large residential complexes assigned the ground floor to the parking make it less exposed to the flood, and the industrial buildings have a relatively high plinth as compared to the small individual houses. Still, most industries have manufacturing units that require heavy instruments operated on the ground floor, which makes them more exposed to the flood.

This study contributed for developing a framework for the flood risk of the municipal council, which has semiurban settlements along with industrial zones and agricultural land. Incorporating the exposure based on the land use and land cover classes makes the framework more effective in calculating the overall flood risk of municipal councils. The study also considered incorporating the corresponding plinth height with 100-year flood depth. The flood risk in urban regions is more complicated than basin-scale flood risk assessment due to diverse socioeconomic conditions, which underestimated the vulnerability. Therefore incorporation of exposure based on industrial zones and infrastructural condition give a better evaluation of flood risk. Further, the flood risk assessment framework can be improved by incorporating the flood preparedness factors like available disaster mitigation resources in each ward, which can ultimately help to understand the allocation of the resources in different parts of the municipal councils.

\section{CONCLUSION}

The study presented a multi-dimensional approach for flood risk assessment. To map flood risk of the unplanned semi-urban region, the socio-economic and medical factors and infrastructural exposure that indicate the probable direct and indirect damages are essential elements. The intensity of the flood risk majorly depends on the possible damages due to flood extent, which can be calculated with factors like population spread across the municipal council and their socio-economic and medical condition. The flood risk mapping helps to understand the high-risk and low-risk zones within the municipal council. The main conclusions of the study are:

- The proposed flood risk assessment framework includes some of the crucial yet neglected indicators like the medical condition of the population to fight against the flu due to flood water and water-borne diseases, which can classify under the flood preparedness of the authorities.

- The ward-wise flood risk maps generated in this study are very useful for the authorities for the disaster funding and resources distribution at ward levels. With such zoning, the authorities can modify the resource distributions in the municipal council.

- Mapping of the flood risk according to the land use and land cover classes shows the monitory damages are different for each class, and the consequences of the risk cannot be defined solely based on the socio-economic vulnerability. For example, the population distribution data considers the residential population, and therefore, the industrial region cannot be included under a high-risk zone.

In the densely populated regions, where the different types of housing are intertwined in a single residential zone it is difficult to map the flood risk. Therefore, the flood exposure based on the different types of housing and their possible damages is important to include in the risk assessment framework.

\section{STATEMENTS AND DECLARATIONS}


FUNDING: The authors declare that no funds, grants, or other support were received during the preparation of this manuscript.

COMPETING INTERST: The authors have no relevant financial or non-financial interests to disclose.

AUTHOR CONTRIBUTION: All authors contributed to the study conception and design. Material preparation, data collection and analysis were performed by Sachin Bhere. The first draft of the manuscript was written by Sachin Bhere and all authors commented on previous versions of the manuscript. All authors read and approved the final manuscript.

\section{REFERENCES}

Ali, Karamat, Roshan M. Bajracharya, and Hriday Lal. Koirala. 2016. "A Review of Flood Risk Assessment." International Journal of Environment, Agriculture and Biotechnology 1(4): 1065-77.

Barrett, Sam, Dave Steinbach, and Simon Addison. 2021. Assessing Vulnerabilities to Disaster Displacement A Good Practice Review.

Bengal, West, Subhankar Chakraborty, and Sutapa Mukhopadhyay. 2019. "Assessing Flood Risk Using Analytical Hierarchy Process ( AHP ) and Geographical Information System ( GIS ): Application." Natural Hazards 99(1): 247-74. https://doi.org/10.1007/s11069-019-03737-7.

Cançado, Vanessa, Lucas Brasil, Nilo Nascimento, and André Guerra. 2008. "Flood Risk Assessment in an Urban Area : Measuring Hazard and Vulnerability.” (January).

Chen, Yi Ru, Chao Hsien Yeh, and Bofu Yu. 2011. "Integrated Application of the Analytic Hierarchy Process and the Geographic Information System for Flood Risk Assessment and Flood Plain Management in Taiwan." Natural Hazards 59(3): 1261-76.

Danumah, Jean Homian et al. 2016. "Flood Risk Assessment and Mapping in Abidjan District Using MultiCriteria Analysis (AHP) Model and Geoinformation Techniques, (Cote d'ivoire)." Geoenvironmental Disasters 3(1). http://dx.doi.org/10.1186/s40677-016-0044-y.

Das, Sumit. 2019a. "Geospatial Mapping of Flood Susceptibility and Hydro-Geomorphic Response to the Floods in Ulhas Basin, India." Remote Sensing Applications: Society and Environment 14(January): 6074. https://doi.org/10.1016/j.rsase.2019.02.006. - 2019b. "Geospatial Mapping of Flood Susceptibility and Hydro-Geomorphic Response to the Floods in Ulhas Basin, India." Remote Sensing Applications: Society and Environment.

Das, Sumit, and Sudhakar D. Pardeshi. 2018. "Morphometric Analysis of Vaitarna and Ulhas River Basins, Maharashtra, India: Using Geospatial Techniques." Applied Water Science 8(6): 1-11. https://doi.org/10.1007/s13201-018-0801-z.

Díez-Herrero, Andrés, and Julio Garrote. 2020. "Flood Risk Assessments: Applications and Uncertainties." Water (Switzerland) 12(8): 1-11.

Doorga, Jay R.S. et al. 2022. "GIS-Based Multi-Criteria Modelling of Flood Risk Susceptibility in Port Louis, Mauritius: Towards Resilient Flood Management.” International Journal of Disaster Risk Reduction 
Feldman, Arlen D. 2000. "Hydrologic Modeling System Technical Reference Manual." Hydrologic Modeling System HEC-HMS Technical Reference Manual (March): 148.

Franci, Francesca et al. 2016. "Satellite Remote Sensing and GIS-Based Multi-Criteria Analysis for Flood Hazard Mapping." Natural Hazards 83(1): 31-51.

Ganguli, Poulomi, and M. Janga Reddy. 2013. "Probabilistic Assessment of Flood Risks Using Trivariate Copulas." Theoretical and Applied Climatology 111(1-2): 341-60.

Ghosh, Abhishek, and Shyamal Kumar Kar. 2018. "Application of Analytical Hierarchy Process (AHP) for Flood Risk Assessment: A Case Study in Malda District of West Bengal, India.” Natural Hazards 94(1): 349-68. https://doi.org/10.1007/s11069-018-3392-y.

Grigg, Neil S. 2020. "Uncertainty and Legal Foreseeability in Flood Risk Management." ASCE-ASME Journal of Risk and Uncertainty in Engineering Systems, Part A: Civil Engineering 6(3): 06020001.

Hu, Shanshan, Xiangjun Cheng, Demin Zhou, and Hong Zhang. 2017. "GIS-Based Flood Risk Assessment in Suburban Areas: A Case Study of the Fangshan District, Beijing." Natural Hazards 87(3): 1525-43.

Imran, Muhammad, Kalsoom Sumra, Syed Amer, and Syed Faisal. 2019. "International Journal of Disaster Risk Reduction Mapping Flood Vulnerability from Socioeconomic Classes and GI Data : Linking Socially Resilient Policies to Geographically Sustainable Neighborhoods Using PLS-SEM.” International Journal of Disaster Risk Reduction 41(July): 101288. https://doi.org/10.1016/j.ijdrr.2019.101288.

Jato-Espino, Daniel, Nora Sillanpää, Ignacio Andrés-Doménech, and Jorge Rodriguez-Hernandez. 2018. "Flood Risk Assessment in Urban Catchments Using Multiple Regression Analysis.” Journal of Water Resources Planning and Management 144(2): 04017085.

Kabenge, Martin, Joshua Elaru, Hongtao Wang, and Fengting Li. 2017. "Characterizing Flood Hazard Risk in Data-Scarce Areas, Using a Remote Sensing and GIS-Based Flood Hazard Index.” Natural Hazards 89(3): 1369-87.

Kim, Sangpil, Hong Gyoo Sohn, Mi Kyeong Kim, and Hyongki Lee. 2019. "Analysis of the Relationship among Flood Severity, Precipitation, and Deforestation in the Tonle Sap Lake Area, Cambodia Using Multi-Sensor Approach.” KSCE Journal of Civil Engineering 23(3): 1330-40.

Kittipongvises, Suthirat et al. 2020. “AHP-GIS Analysis for Flood Hazard Assessment of the Communities Nearby the World Heritage Site on Ayutthaya Island, Thailand.” International Journal of Disaster Risk Reduction 48(April): 101612. https://doi.org/10.1016/j.ijdrr.2020.101612.

Koks, E. E., M. Bočkarjova, H. de Moel, and J. C.J.H. Aerts. 2015. "Integrated Direct and Indirect Flood Risk Modeling: Development and Sensitivity Analysis." Risk Analysis 35(5): 882-900.

Koks, E E, B Jongman, T G Husby, and W J W Botzen. 2014. "ScienceDirect Combining Hazard, Exposure and Social Vulnerability to Provide Lessons for Flood Risk Management.” Environmental Science and 
582

583

584

585

586

587

588

589

590

591

592

593

594

595

596

597

598

599

600

601

602

603

604

605

606

607

608

609

610

611

612

613

614

Komolafe, Akinola Adesuji, Srikantha Herath, and Ram Avtar. 2018. "Methodology to Assess Potential Flood Damages in Urban Areas under the Influence of Climate Change.” Natural Hazards Review 19(2): 05018001 .

Kothyari, Umesh C., and Ramchandra J. Garde. 1992. "Rainfall Intensity-Duration-Frequency Formula for India.” Journal of Hydraulic Engineering 118(2): 323-36.

Kron, Wolfgang. 2002. "Keynote Lecture : Flood Risk = Hazard $\times$ Exposure $\times$ Vulnerability." : 82-97.

Lin, Lin, Zening Wu, and Qiuhua Liang. 2019. "Urban Flood Susceptibility Analysis Using a GIS-Based MultiCriteria Analysis Framework.” Natural Hazards 97(2): 455-75. https://doi.org/10.1007/s11069-01903615-2.

Merz, B, H Kreibich, R Schwarze, and A Thieken. 2010. "Review Article ‘ Assessment of Economic Flood Damage ." : 1697-1724.

Mohanty, Mohit Prakash, Sahil Mudgil, and Subhankar Karmakar. 2020. "Flood Management in India: A Focussed Review on the Current Status and Future Challenges." International Journal of Disaster Risk Reduction 49(March): 101660. https://doi.org/10.1016/j.ijdrr.2020.101660.

Mokhtari, Fahimeh, Saeid Soltani, and Seyed Alireza Mousavi. 2017. “ Assessment of Flood Damage on Humans, Infrastructure, and Agriculture in the Ghamsar Watershed Using HEC-FIA Software ." Natural Hazards Review 18(3): 04017006.

Molinari, Daniela et al. 2019. "Validation of Flood Risk Models: Current Practice and Possible Improvements." International Journal of Disaster Risk Reduction 33(May 2018): 441-48. https://doi.org/10.1016/j.ijdrr.2018.10.022.

Monteil, Charlotte et al. 2022. "Rethinking the Share of Responsibilities in Disaster Preparedness to Encourage Individual Preparedness for Flash Floods in Urban Areas.” International Journal of Disaster Risk Reduction 67(July 2021).

Moore, I. D., R. B. Grayson, and A. R. Ladson. 1991. “Digital Terrain Modelling: A Review of Hydrological, Geomorphological, and Biological Applications." Hydrological Processes 5(1): 3-30.

Nguyen, Huu Duy et al. 2021. "Predicting Future Urban Flood Risk Using Land Change and Hydraulic Modeling in a River Watershed in the Central Province of Vietnam.” Remote Sensing 13(2): 1-24.

Nofal, Omar M., and John W. van de Lindt. 2020. "Probabilistic Flood Loss Assessment at the Community Scale: Case Study of 2016 Flooding in Lumberton, North Carolina.” ASCE-ASME Journal of Risk and Uncertainty in Engineering Systems, Part A: Civil Engineering 6(2): 05020001.

Ramkar, Preeti, and Sanjaykumar M. Yadav. 2021. "Flood Risk Index in Data-Scarce River Basins Using the AHP and GIS Approach.” Natural Hazards 109(1): 1119-40. https://doi.org/10.1007/s11069-021-04871$\mathrm{X}$. 
Rangari, Vinay Ashok, V. Sridhar, N. V. Umamahesh, and Ajey Kumar Patel. 2019. "Floodplain Mapping and Management of Urban Catchment Using HEC-RAS: A Case Study of Hyderabad City." Journal of The Institution of Engineers (India): Series A 100(1).

Saaty, R. W. 1987. “The Analytic Hierarchy Process-What It Is and How It Is Used.” Mathematical Modelling 9(3-5): 161-76.

Sahoo, Sanat Nalini, and Pekkat Sreeja. 2017. "Development of Flood Inundation Maps and Quantification of Flood Risk in an Urban Catchment of Brahmaputra River.” ASCE-ASME Journal of Risk and Uncertainty in Engineering Systems, Part A: Civil Engineering 3(1): 1-11.

Sen, Mrinal Kanti, Subhrajit Dutta, and Jahir Iqbal Laskar. 2021. “A Hierarchical Bayesian Network Model for Flood Resilience Quantification of Housing Infrastructure Systems.” ASCE-ASME Journal of Risk and Uncertainty in Engineering Systems, Part A: Civil Engineering 7(1): 04020060.

Stevens, Forrest R., Andrea E. Gaughan, Catherine Linard, and Andrew J. Tatem. 2015. "Disaggregating Census Data for Population Mapping Using Random Forests with Remotely-Sensed and Ancillary Data.” PLoS ONE 10(2): 1-22.

Svetlana, Dobrovičová, Dobrovič Radovan, and Dobrovič Ján. 2015. “The Economic Impact of Floods and Their Importance in Different Regions of the World with Emphasis on Europe." Procedia Economics and Finance 34(15): 649-55.

Tripathy, Shrabani S., Hari Vittal, Subhankar Karmakar, and Subimal Ghosh. 2020a. "Flood Risk Forecasting at Weather to Medium Range Incorporating Weather Model, Topography, Socio-Economic Information and Land Use Exposure.” Advances in Water Resources 146(April): 103785. https://doi.org/10.1016/j.advwatres.2020.103785.

Tripathy, Shrabani S, Hari Vittal, Subhankar Karmakar, and Subimal Ghosh. 2020b. “Advances in Water Resources Flood Risk Forecasting at Weather to Medium Range Incorporating Weather Model , Topography, Socio-Economic Information and Land Use Exposure.” Advances in Water Resources 146(October): 103785. https://doi.org/10.1016/j.advwatres.2020.103785.

USACE. 2016. "HEC-RAS River Analysis System Hydraulic Reference Manual Version 5.0.” Hydrologic Engineering Center (February): 547.

Utazi, C. Edson et al. 2018. "High Resolution Age-Structured Mapping of Childhood Vaccination Coverage in Low and Middle Income Countries." Vaccine 36(12): 1583-91. https://doi.org/10.1016/j.vaccine.2018.02.020.

Wang, Yi et al. 2019. “A Hybrid GIS Multi-Criteria Decision-Making Method for Flood Susceptibility Mapping at Shangyou, China." Remote Sensing 11(1). 
Figures

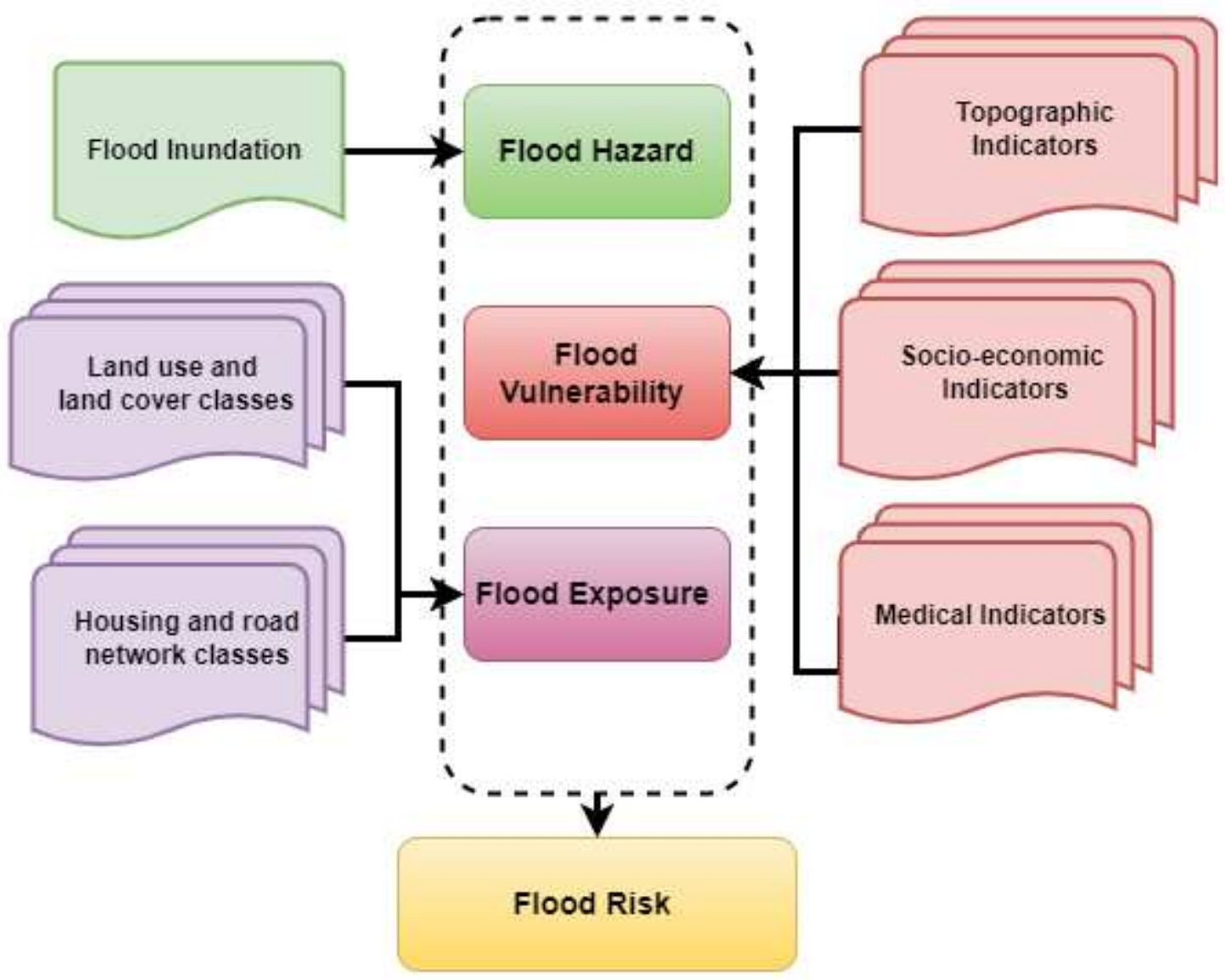

Figure 1

Flow chart depicting different steps involved for flood risk assessment. 


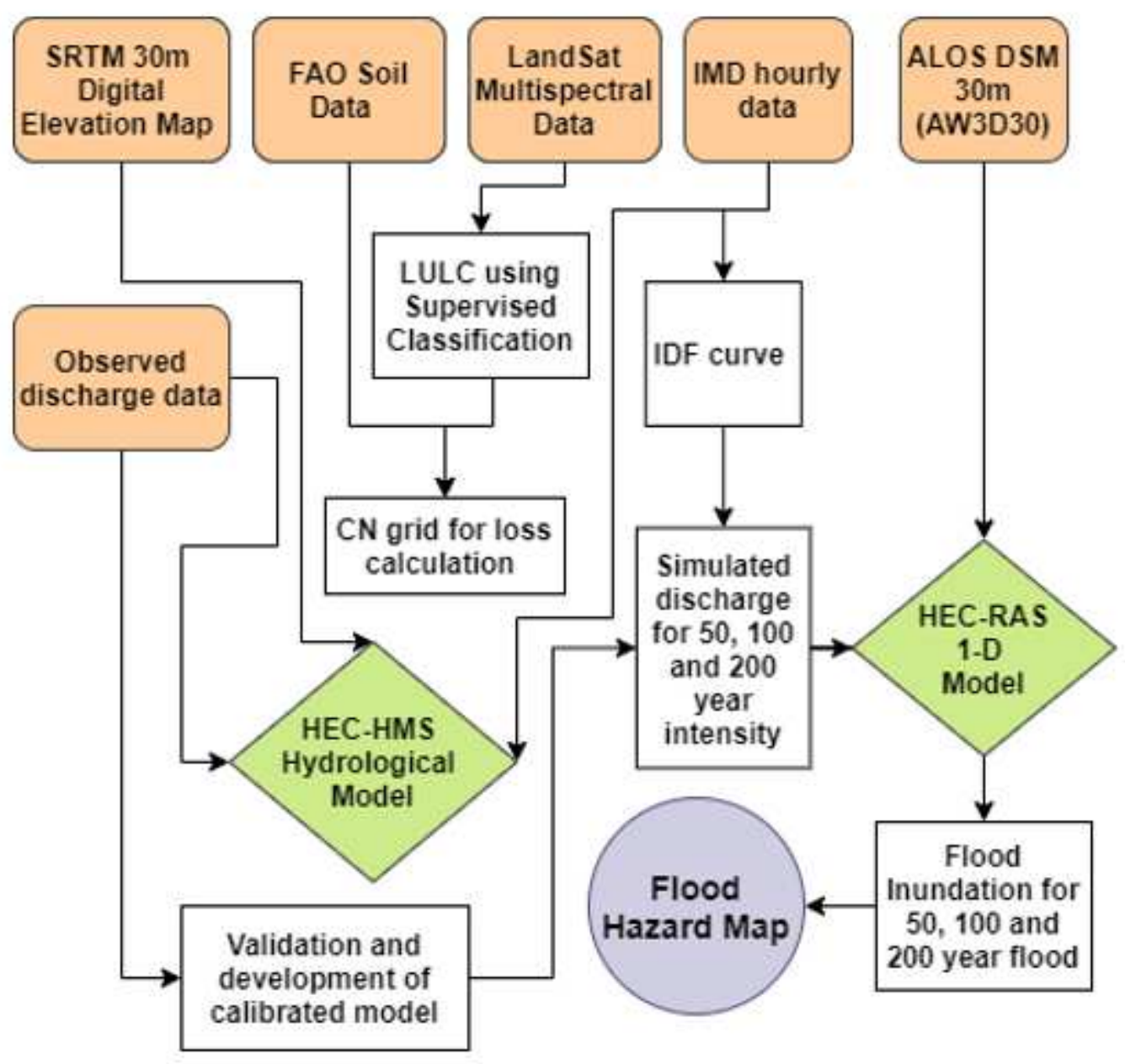

Figure 2

Flow chart depicting different steps involved for flood hazard mapping. The flow chart includes the input data for calculating the discharge for different return periods using HEC-HMS model and then the extent of flood inundation using the HEC-RAS model. 


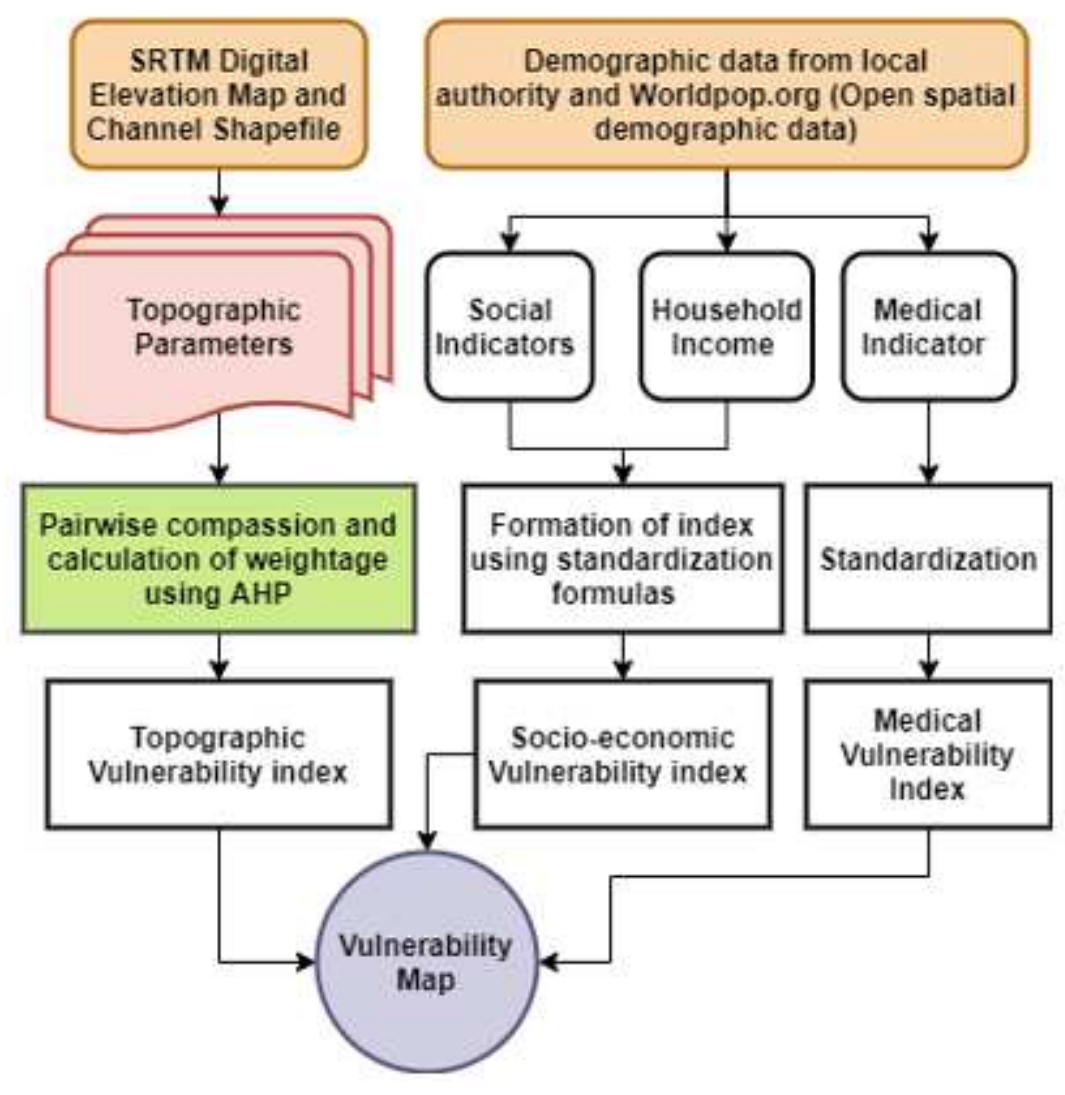

Figure 3

Flow chart depicting different steps involved for flood Vulnerability mapping. The vulnerability is calculated by considering topographic vulnerability and socio-economic vulnerability.

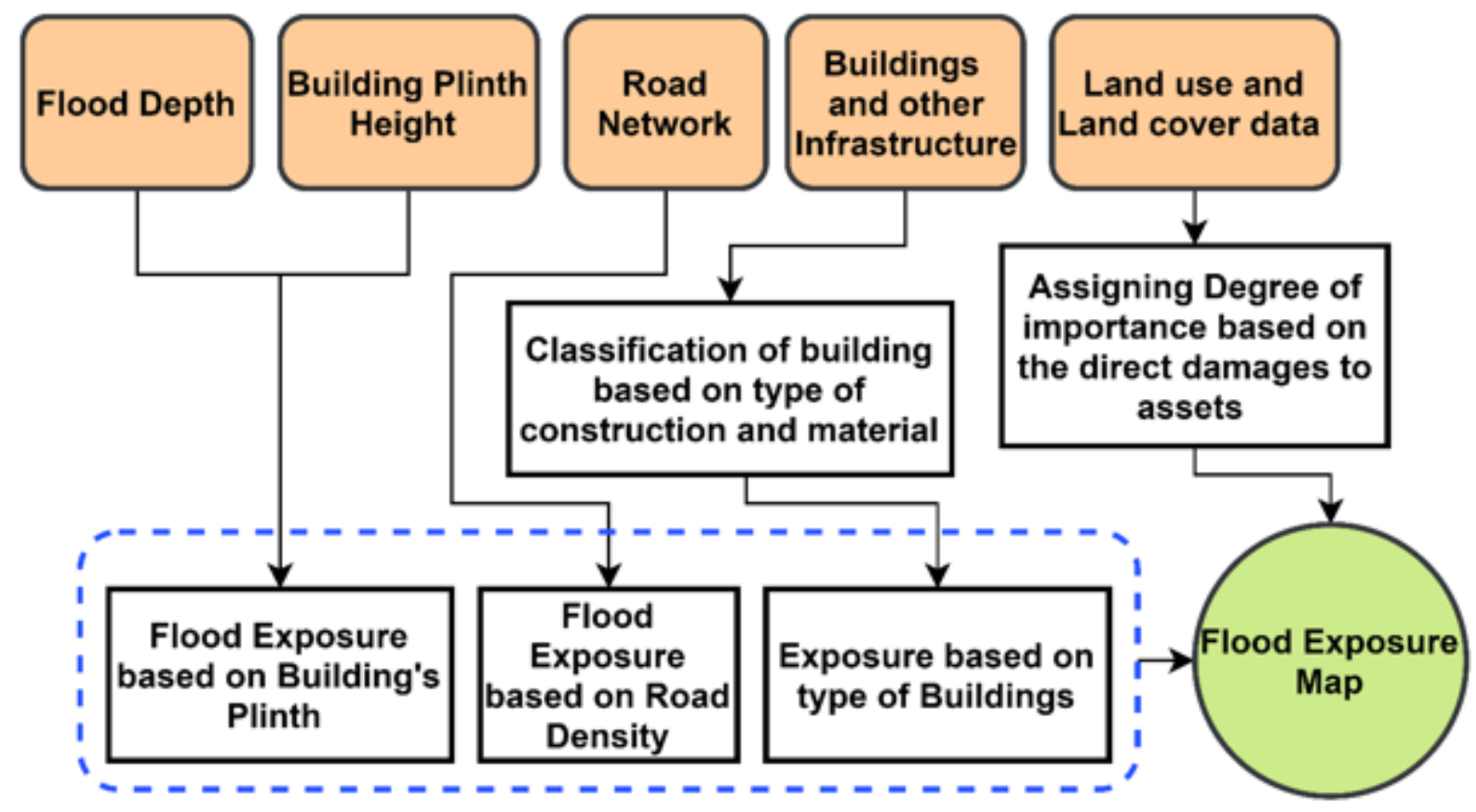




\section{Figure 4}

The flowchart depicting the steps for calculating the flood exposure. The exposure is calculated by considering building and infrastructure exposure, and land use and land cover exposure.

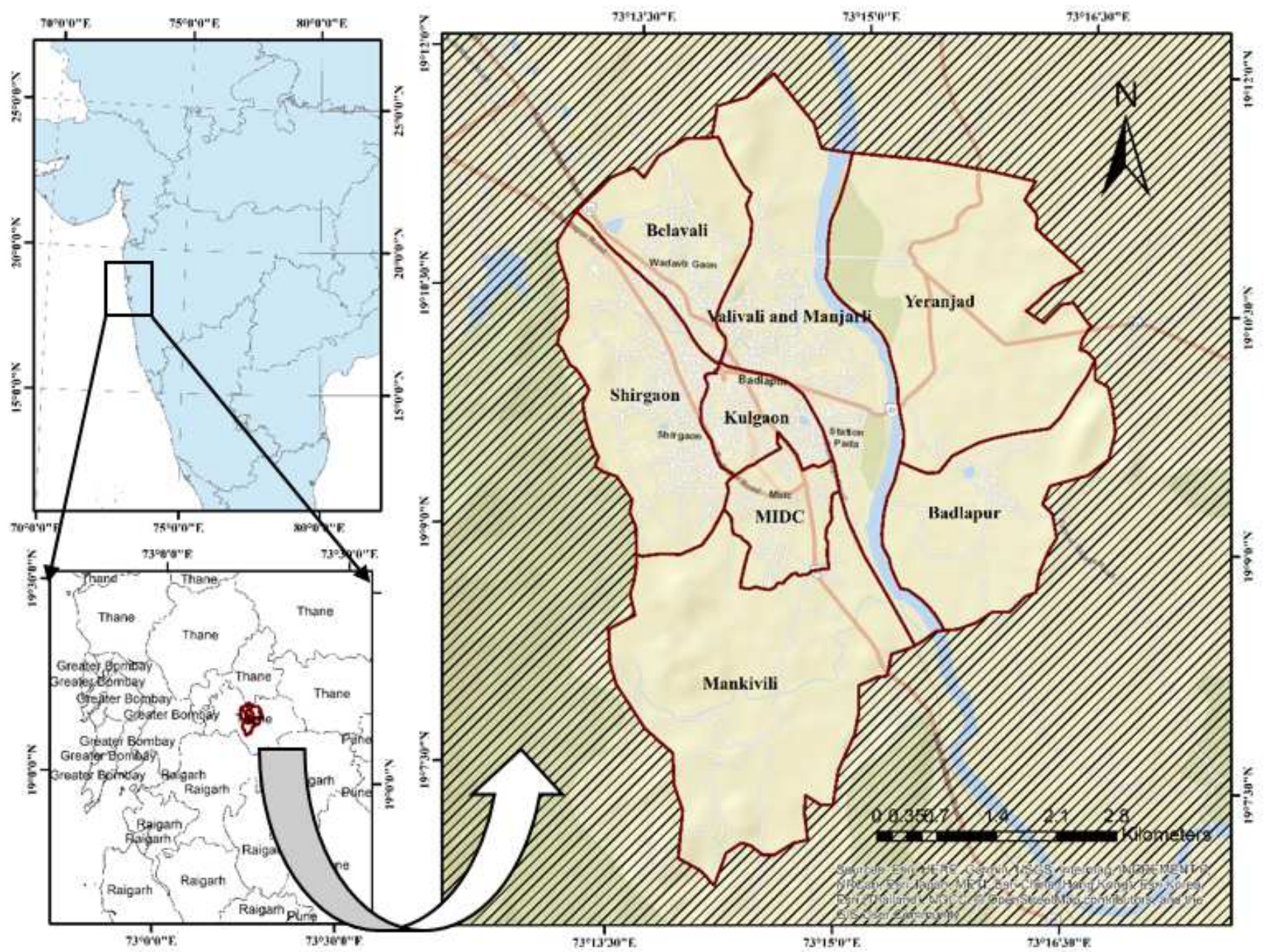

Figure 5

Location map of Kulgoan-Badlapur Municipal Council's (KBMC) area. 


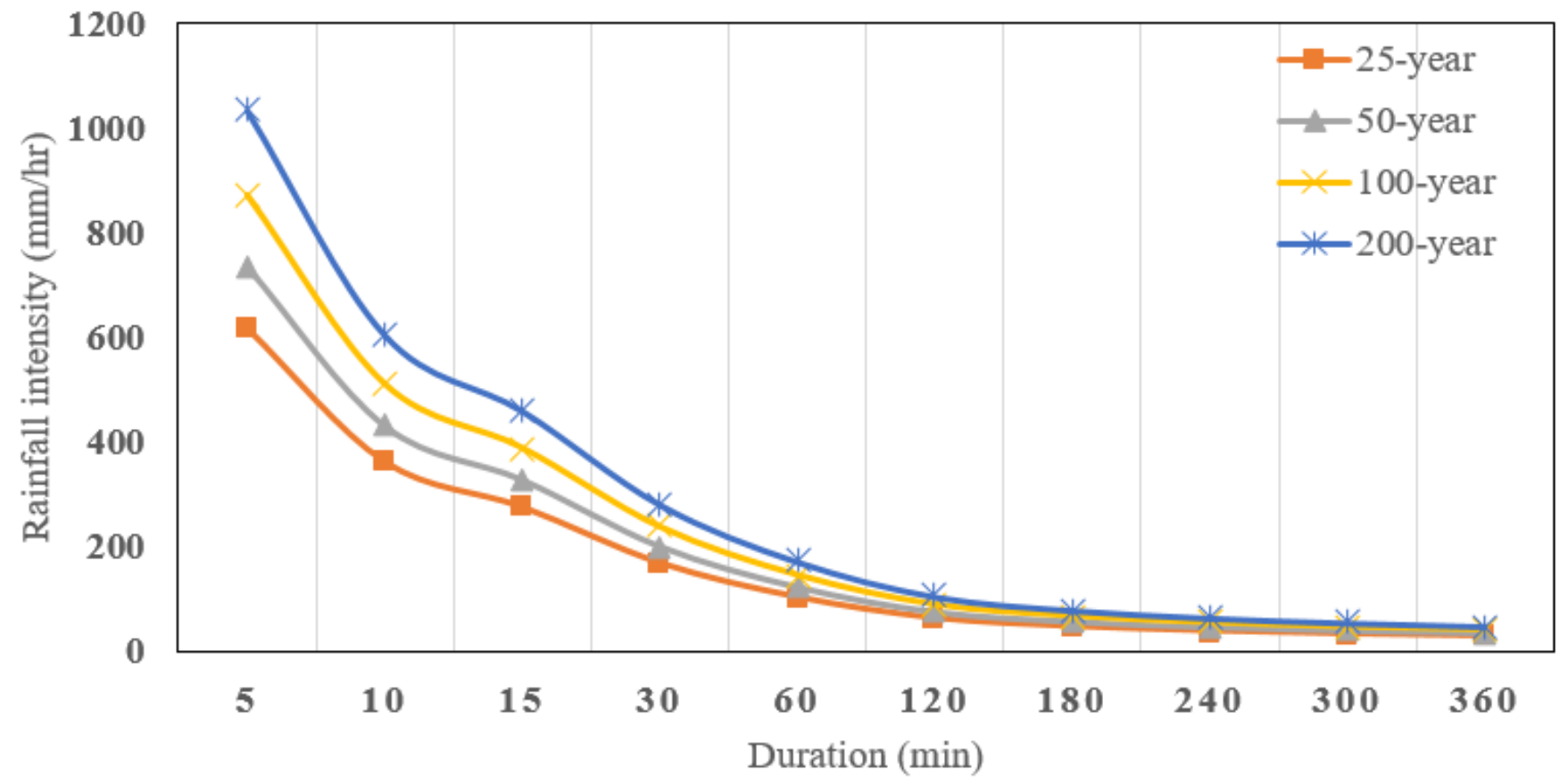

Figure 6

Intensity-Duration-Frequency (IDF) curves derived for Kulgaon-Badlapur Municipal Council (KBMC) area. The graph shows the intensity of the rainfall in $\mathrm{mm} / \mathrm{hr}$ for 25-year, 50-year, 100-year, and 200-year return periods. 


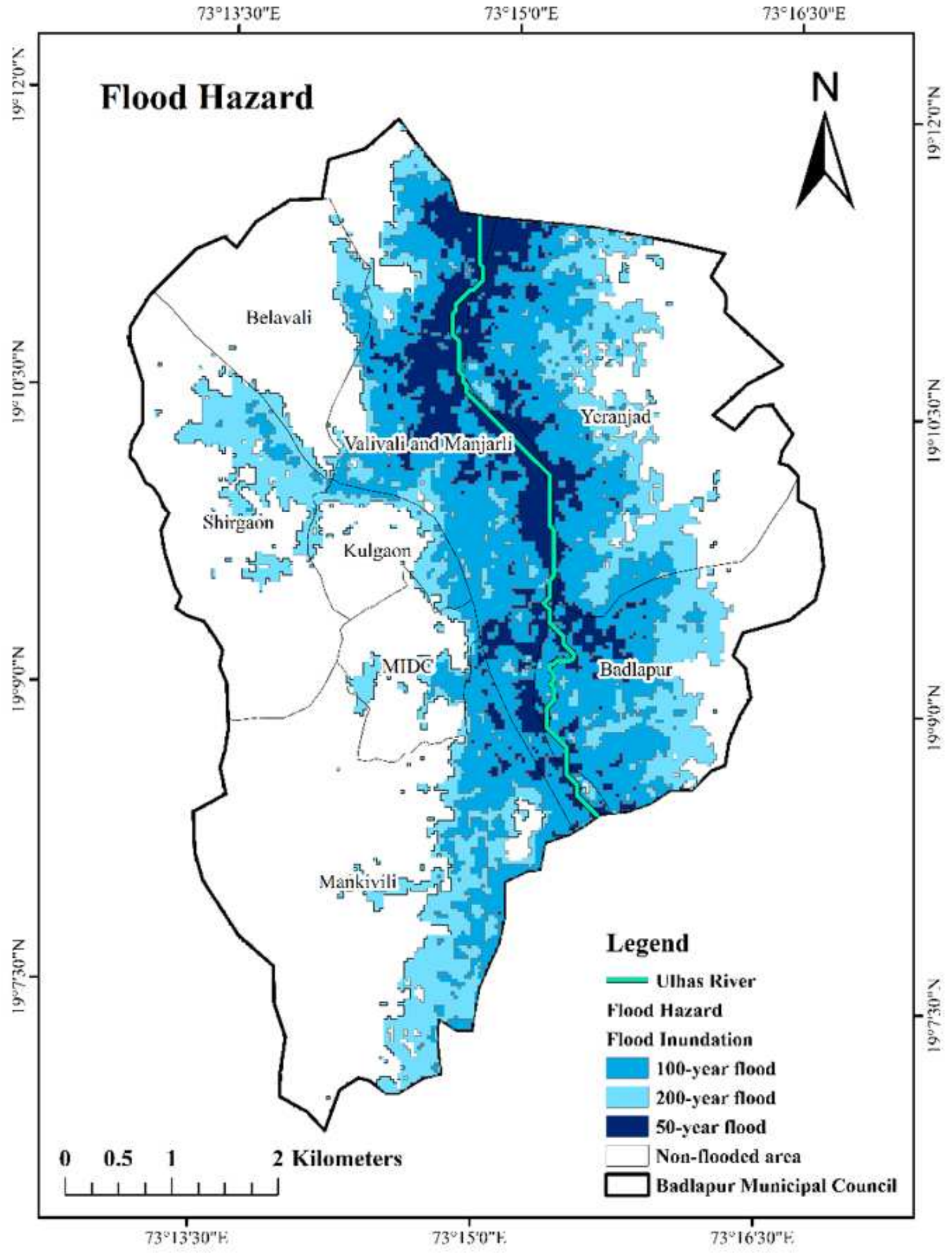

Figure 7

Flood hazard map for Kulgaon-Badlapur Municipal Council (KBMC). The flood hazard is calculated by determining the flood inundation associated with 50-year, 100-year and 200-year return periods. 


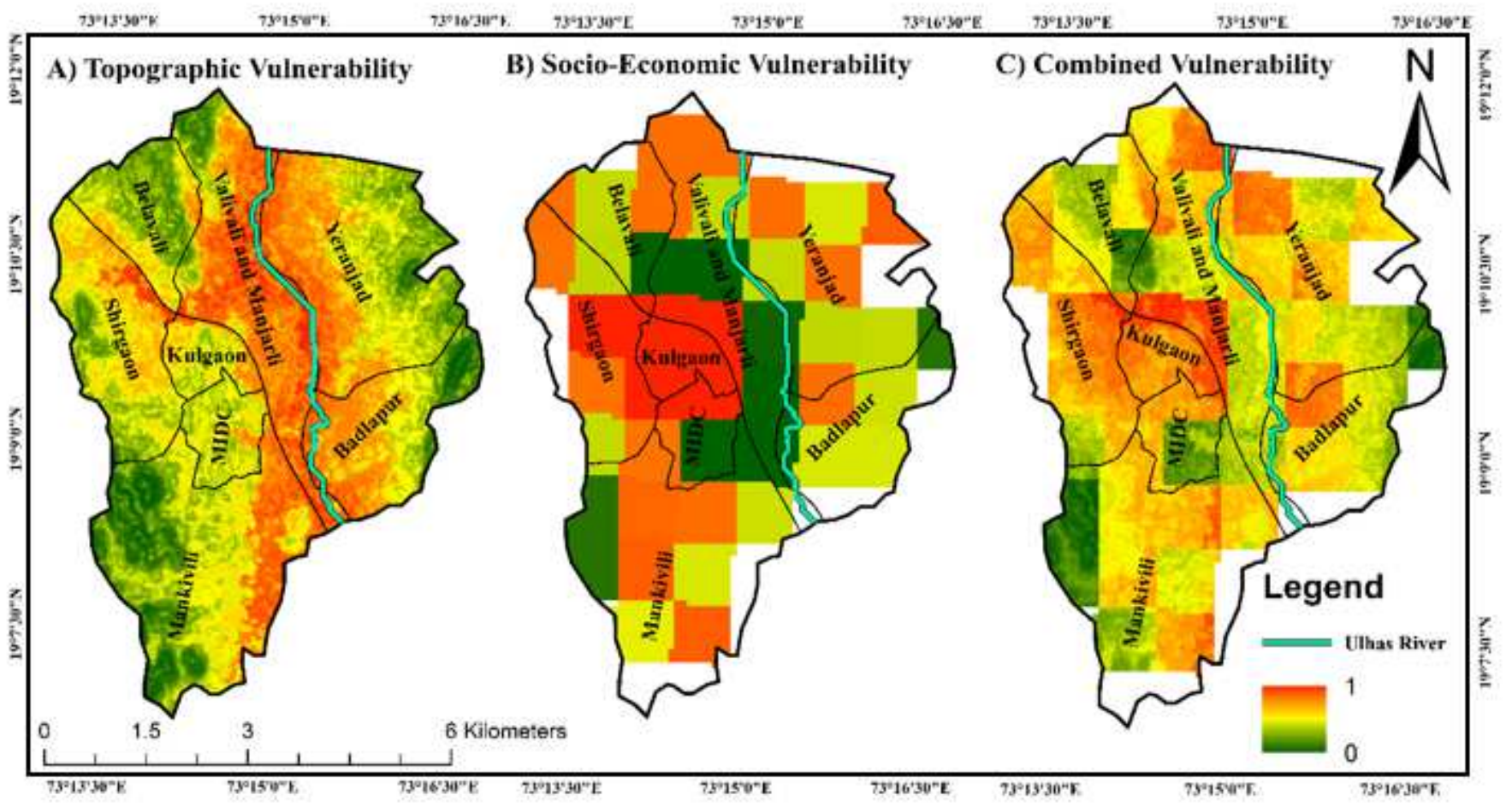

Figure 8

The maps showing spatial distribution of vulnerability in Kulgaon Badlapur Municipal Council (KBMC) area: (A) Topographic vulnerability map; (B) The socio-economic vulnerability map, obtained by combining the social, economic, and medical indicators; (C) The combined flood vulnerability map, obtained by combining the topographic and socio-economic vulnerability.

\section{Figure 9}

The flood exposure maps for the Kulgaon-Badlapur Municipal Council (KBMC) area, obtained by considering $(A)$ the direct damages based on the land use and land cover classes; (B) based on the road network; $(C)$ The flood exposure map, based on the combination of infrastructural exposure which is calculated by considering road density, plinth height, and type of building along with a degree of importance assigned to each land use and land cover class based on the probable direct damages. 


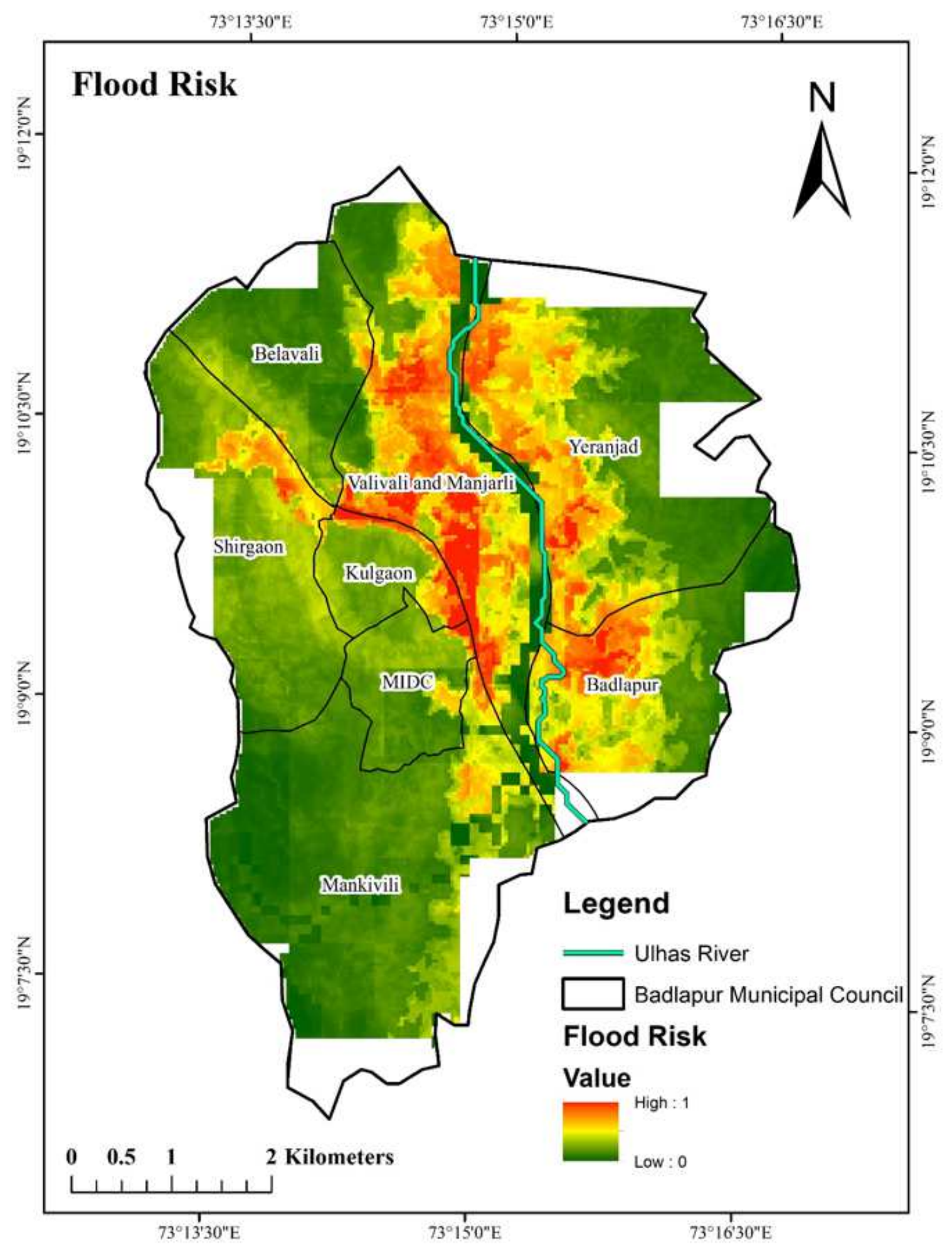

Figure 10

The flood risk map of Kulgaon-Badlapur Municipal council (KBMC) area. The risk is calculated by multiplying the flood hazard, vulnerability, and exposure. 